\title{
Intravenous methylcobalamin effectively ameliorated painful diabetic neuropathy: A randomized double-blind placebo controlled trial
}

\author{
Thomas Eko Purwata', I Putu Eka Widyadharma', Made Rudy², Andreas Soejitno³ \\ Wira Gotera ${ }^{4}$, Ketut Suryana ${ }^{5}$ \\ ${ }^{1}$ Division of Pain, Department of Neurology, Faculty of Medicine, Udayana University/Sanglah Hospital, Bali, Indonesia \\ ${ }^{2}$ Department of Neurology, Siloam Hospital, Bali, Indonesia \\ ${ }^{3}$ Faculty of Medicine, Udayana University/Sanglah Hospital, Bali, Indonesia \\ ${ }^{4}$ Division of Endocrinology, Department of Internal Medicine, Faculty of Medicine, Udayana University/Sanglah Hospital, \\ Bali, Indonesia \\ ${ }^{5}$ Department of Internal Medicine, Wangaya Hospital, Bali, Indonesia
}

Objective. Painful diabetic neuropathy (PDN) is a prevalent debilitating consequence of diabetes mellitus with lack of satisfactory therapeutic options. Methylcobalamin (MeCbl) is one of vitamin B12 analogs with known neurotrophic effects. We aimed to determine if MeCbl can relieve PDN.

Materials and methods. This was a randomized (1:1) double-blind placebo-controlled trial involving PDN patients. Treatment and control group received daily $12.5 \mathrm{mg}$ oral amitryptiline bid with either $500 \mu \mathrm{g}$ of intravenous MeCbl or saline injection given on alternating days, respectively, for a 9-consecutive day period. PDN was assessed with douleur neuropathique 4 (DN4) questionnaire. Numeric pain rating scale (NPRS) was used to monitor pain intensity and treatment response. All investigators and patients were kept blinded throughout the study period.

Outcomes. 42 patients, 21 on each arm had completed the study. The NPRS reduction can already be observed as early as day 2 post-intervention. Both the treatment and control group demonstrated sustained reduction of NPRS by almost one point per each time point of evaluation in the first three days $(p<0.001)$. NPRS reduction remained until the end of the study period. The treatment group had a significantly lower NPRS score by 1.29 than that of the control group during the entire study period $(95 \% \mathrm{Cl}-1.84--0.75 ; \mathrm{p}<0.001)$. Treatment group experienced significantly higher NPRS reduction when compared with control (4.19 \pm 1.54 vs. $2.1 \pm 0.83 ; 95 \% \mathrm{Cl} 1.32-2.87 ; p<0.001)$, i.e. $62.6 \%$ from baseline.

Conclusions. MeCbl significantly and safely relieved PDN in a relatively rapid onset.

Keywords: methylcobalamin, painful diabetic neuropathy, efficacy, intravenous, adjuvant

\section{INTRODUCTION}

PDN is one of the complications of diabetes mellitus (DM) which consists of various painful sensation (burning, painful cold, electric shocks) as a result of diffuse damage to the peripheral nerve fibers and its consequent peripheral nerve dysfunction $[1,2]$. PDN is very common that it can affect one in five DM patients [3]. The painful symptoms are often debilitating, impairing patient's functional performance and daily activity, associated with higher rate of depression and anxiety, poses significant financial burden, and thus can be catastrophic to the patients, caregivers, and countries by reducing patient's life expectancy, quality of life, and productiv- 
ity [1,3-6]. To date, there has been limited treatment modalities with high efficacy and sustainable improvement in PDN.

Methylcobalamin (MeCbl) is one of the many vitamin B12 analogs, in which it differs from cyanocobalamin by the replacement of cyanide with methyl group $\left(\mathrm{CH}_{3}-\mathrm{B} 12\right)$ [7]. $\mathrm{MeCbl}$ is considered the best form of vitamin B12 for nerve because of its high concentration in the CSF (constituting 90\% of total cobalamin in the CSF) and higher uptake by neuronal organelles [8,9]. MeCbl ameliorates neuropathic pain via multiple mechanisms, including promoting axonal regeneration, protecting against glutamate-induced neurotoxicity, and inhibiting ectopic spontaneous discharge $[7,10,11]$. In fact, $\mathrm{MeC}-$ bl has been effective in ameliorating neuropathic pain from various pathologies, including sciatica, herpetic, glossopharyngeal, and trigeminal neuralgia [7]. However, $\mathrm{MeCbl}$ for the treatment of $\mathrm{DN}$ has been studied in multiple trials with mixed results, and only a few specifically targeted PDN $[8,12,13]$.

\section{AIM}

Herein we would like to evaluate the efficacy and safety profile of MeCbl in the treatment of PDN.

\section{MATERIALS AND METHODS}

\section{Study design}

This was a randomized, double-blind, placebo-controlled trial in patients with diabetes and painful diabetic neuropathy. Study reporting was based on CONSORT guidelines [14]. The study was conducted between January and June 2020. The primary outcome of the study was to determine the rate of NPRS reduction between groups, before and after an intervention, whereas the secondary outcome was to determine the onset of NPRS reduction and its sustainability over time. Patients were recruited from two hospitals in Denpasar, i.e. Sanglah and Wangaya hospital, either admitted via diabetic center or neurology clinic. All patients must met the inclusion criteria by which they were diagnosed with type 2 diabetes mellitus by a physician and suffered from diabetic neuropathy as confirmed by DN4 questionnaire score of greater than or equal to 4 , assessed by neurologists blinded to patient's intervention status [15].

Exclusion criteria comprised patients with chronic liver and/or kidney disease, suffered from mild pain, patients with known HIV infection or leprosy or malignancy-induced neuropathy, patients who had already suffered from neuropathic pain and treated with antiretroviral or chemotherapeutic agents, analgesics (either for nociceptive or neuropathic pain, or both, including NSAIDs, GABA an- alogues, tricyclic antidepressasnts, serotonin norepinephrine reuptake inhibitors, opioid, or topical agents), vitamin B, or estrogen, patients with history of significant exposure to alcohol (defined as more than one and two standard drinks per day for women and men, respectively within the last one year), pesticide, mercury, organophosphate, and lead, patients with carpal tunnel syndrome and/or cervical root syndrome, patients with moderate to extreme depression as marked by Beck Depression Inventory score of greater than or equal to 21 , and those with contraindication or previously known adverse drug reactions to either amytriptiline or $\mathrm{MeCbl}$, or both. All included patients were willing to participate in the study and provided written informed consent. The study protocol was approved by the Ethical Commission for Research Faculty of Medicine Udayana University/Sanglah Hospital with protocol no. of 920.02.1 under ethical clearance no. 10/UN.14.2./Litbang and adhered to the WMA Declaration of Helsinki of ethical principles for medical research involving human subjects.

\section{Treatments}

All eligible patients were subsequently randomized by 1:1 ratio using computer-based random number generator into either treatment or control group. Both members of the group were interviewed to obtain baseline numerical pain rating scale (NPRS). Afterward, the treatment group received daily oral amitriptyline $12.5 \mathrm{mg}$ bid with 500 $\mu \mathrm{g}(1 \mathrm{ml})$ qd of intravenous $\mathrm{MeCbl}$ (Methycobal ${ }^{\circledR}$, Eisai). On the other hand, those in the control group received amitryptiline with the same dose and administration as those in the treatment group, accompanied with $1 \mathrm{ml}$ of intravenous saline injection. Oral amitryptiline was consumed daily, whereas both intravenous injections were given on alternating days (i.e. one day on and off) for 10 days (5 total injections on day 1, 3, 5, 7, and 9). Subjects with persistent or worsening neuropathic pain who required an increase of amitryptiline dose were counted as drop out and subsequently treated as a regular patient with eligibility to receive dose increment or additional neuropathic pain medications. $\mathrm{MeCbl}$ and saline solution was allocated into a whole black plaster-sealed syringe with double confirmation check by another independent operator to ensure that there was no visible color can be seen before, during, and after the injection. The doctors who examined the patients for eligibility criteria, obtained DN4 and NPRS score, and administered the injection were kept blinded to the type of intervention given to the patients. Blinding fashion was also applied to all patients. 


\section{Evaluation}

The mean outcome of this study was the reduction of baseline NPRS vs. NPRS after intervention and its magnitude when compared between treatment and control group. Whereas the secondary outcome of this study was the onset of NPRS reduction over time and its significance. All data, including any side effects were routinely recorded during the injection administration, i.e. on day 2, 3, 5, 7, and 9. In addition, we interviewed the patients at one week after completion of the study to obtain the NPRS. Patients were asked for NPRS before the injection procedure. Any serious side effects including (but not limited to) arrhythmia, chest pain, dyspenea, or hypersensitivity to one or both of the medications were immediately reported to the investigators and the corresponding patient was terminated from the study. The physician examined the patients before, during, and at the end of the study. All serious side effects were recorded into the computer database and a proportional difference of $>5 \%$ between the treatment and control group was deemed sufficient to prematurely terminate the study.

\section{Statistical analysis}

All data were initially assessed for normality of distribution. Categorical and interval data pertaining to baseline characteristics between groups were evaluated using chi square and independent $t$ test, respectively. Mean NPRS difference was assessed using repeated measures ANOVA with multiple time periods and intervention types as within-subject factors. In addition, a separate analysis of NPRS reduction before and after intervention among groups were conducted using independent $t$ test with results compared to former analyses. Interval data was expressed as mean \pm SD whenever it was normally distributed, or otherwise stated as median \pm range. A $p$ value of $<0.05$ was regarded as statistically significant. The study was carried out on the basis of intention-to-treat analysis. All statistical analyses were performed by using IBM SPSS Statistics version 20 .

\section{RESULTS}

42 patients were recruited in this study with 21 patients in each group (see study flow chart in Figure 1). All of them completed the study with none being dropped out. Female predominated the study with 57 and $62 \%$ of them in the control and treatment, group respectively. The average duration of diabetes was approximately 4 years with a median of 2 years for the duration of painful diabetic neuropathy across all groups. The majority of patients in the treatment group was on insulin therapy, whereas the proportion of those with oral hypoglycemic drugs and insulin in the control group was comparable. In addition, the blood glucose levels before and after trial intervention were similar across all groups.

The complete description of patient's baseline characteristics can be seen in Table 1. NPRS was recorded at baseline, day 2, 3, 5, 7, 9, and one week after the study had been ended. Mean NPRS at baseline was similar between treatment and placebo groups ( $6.57 \pm 0.28$ and $6.57 \pm 0.22$, respectively) (Table 1). However, treatment group NPRS reduction can be observed as early as day 2 of intervention by more than one point and constantly decreased until day 9. Meanwhile placebo group also demonstrated mean NPRS reduction, albeit lagged behind and to a lesser extent than that of treatment group.

Furthermore, we would like to know the magnitude of NPRS change over time. Repeated measures ANOVA showed NPRS decline as early as day 2 and 3 among treatment $(-1.48$; 95\% CI $-2.36--0.59$; $\mathrm{p}<0.0001)$ and placebo (-1.10; 95\% CI $-1.68--0.51 ; \mathrm{p}$ $<0.0001$ ) group, respectively (Figure 2). In general, NPRS of treatment and placebo continued to decline significantly with a rate of 0.4 to 0.8 and 0.14 to 0.4 , respectively for each day thereafter, only to slow down at the end of the study (i.e. one week post injection vs. day 9). Overall, the rate of NPRS decline on treatment group seemed to be more consistent by means of magnitude and statistical significance when compared with placebo. At the end of the study, treatment group experienced more than 4-point decline of NPRS (-4.24; 95\% CI -5.36 - -0.12; p $<0.0001$ ), twice as much when compared with placebo (-2.10; 95\% CI -2.73 - -1.47; $\mathrm{p}<0.0001)$.

Lastly, we would like to determine the overall magnitude of NPRS change between treatment and control group over time. We found that treatment group had a significantly lower NPRS score by 1.29 than that of control group during the entire study period, i.e. until one week post intervention (95\% CI $-1.84--0.75 ; \mathrm{p}<0.001$ ) (Table 2). In addition, we also performed an independent statistical analysis to assess the net result of NPRS reduction before and after the intervention. The treatment group demonstrated twice NPRS reduction when compared with control group with statistical significance (4- vs. 2-point reduction, respectively; $\mathrm{p}<0.001$ ). Intervention with $\mathrm{MeCbl}$ and amitryptiline was proven to reduce baseline NPRS by $62.6 \%$ as opposed to the relatively modest decline of those who receive saline injection and amitryptiline (31.4\%). All reductions across all groups were statistically significant. 


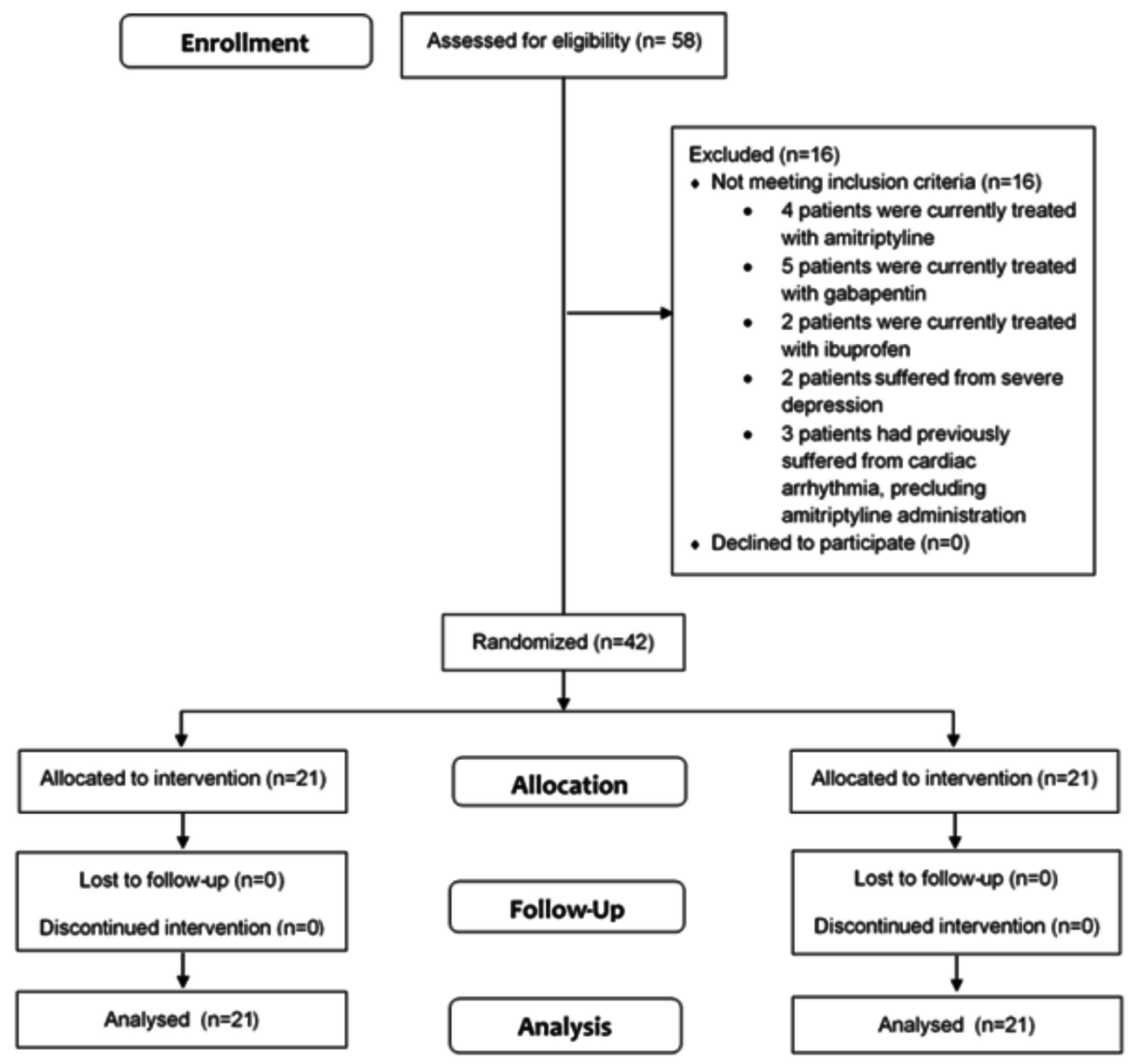

FIGURE 1. Study flowchart

TABLE 1. Baseline characteristics of patients within each group

\begin{tabular}{|c|c|c|c|}
\hline Parameters & Treatment $(n=21)$ & Control (n=21) & $P$ value \\
\hline Male & 8 & 9 & 0.75 \\
\hline Female & 13 & 12 & 0.75 \\
\hline Age (mean $\pm S D)$ & $56.2 \pm 7$ & $59.5 \pm 7.2$ & 0.15 \\
\hline \multicolumn{3}{|l|}{ Education level (\%) } & \multirow{6}{*}{0.06} \\
\hline Illiterate & $0(0)$ & $1(4.8)$ & \\
\hline Elementary & $2(9.5)$ & $2(9.5)$ & \\
\hline Junior high school & $5(23.8)$ & $2(9.5)$ & \\
\hline Senior high school & $6(28.6)$ & $14(66.7)$ & \\
\hline University/diploma & $8(38.1)$ & $2(9.5)$ & \\
\hline Duration of diabetes $(\mathrm{y})$ [mean \pm SD] & $4.2 \pm 1.9$ & $4.2 \pm 1.4$ & 0.51 \\
\hline Random blood glucose before intervention (mg/dL) [mean $\pm S D]$ & $201 \pm 53.4$ & $229.2 \pm 85.9$ & 0.21 \\
\hline Random blood glucose after intervention (mg/dL) [mean $\pm S D$ ] & $176.4 \pm 40.6$ & $198.3 \pm 51.8$ & 0.14 \\
\hline \multicolumn{4}{|l|}{ Type of treatment } \\
\hline Oral hypoglycemic drugs & $5(23.8)$ & $10(47.6)$ & 0.09 \\
\hline Insulin & $16(76.2)$ & $11(52.4)$ & 0.20 \\
\hline Duration of painful diabetic neuropathy $(\mathrm{y})$ [median \pm range] & $2(4)$ & $2(4)$ & 0.84 \\
\hline Systolic blood pressure [median \pm range] & $130(20)$ & $120(40)$ & 0.99 \\
\hline Diastolic blood pressure [median \pm range] & $80(20)$ & $80(20)$ & 0.39 \\
\hline Body mass index $\left(\mathrm{kg} / \mathrm{m}^{2}\right)$ [mean $\left.\pm \mathrm{SD}\right]$ & $23.7 \pm 2.6$ & $23.8 \pm 1.9$ & 0.88 \\
\hline DN4 score [median \pm range] & $5(3)$ & $5(4)$ & 0.50 \\
\hline Baseline NPRS [mean $\pm S D$ ] & $6.57 \pm 1.29$ & $6.57 \pm 1.03$ & 1.00 \\
\hline
\end{tabular}




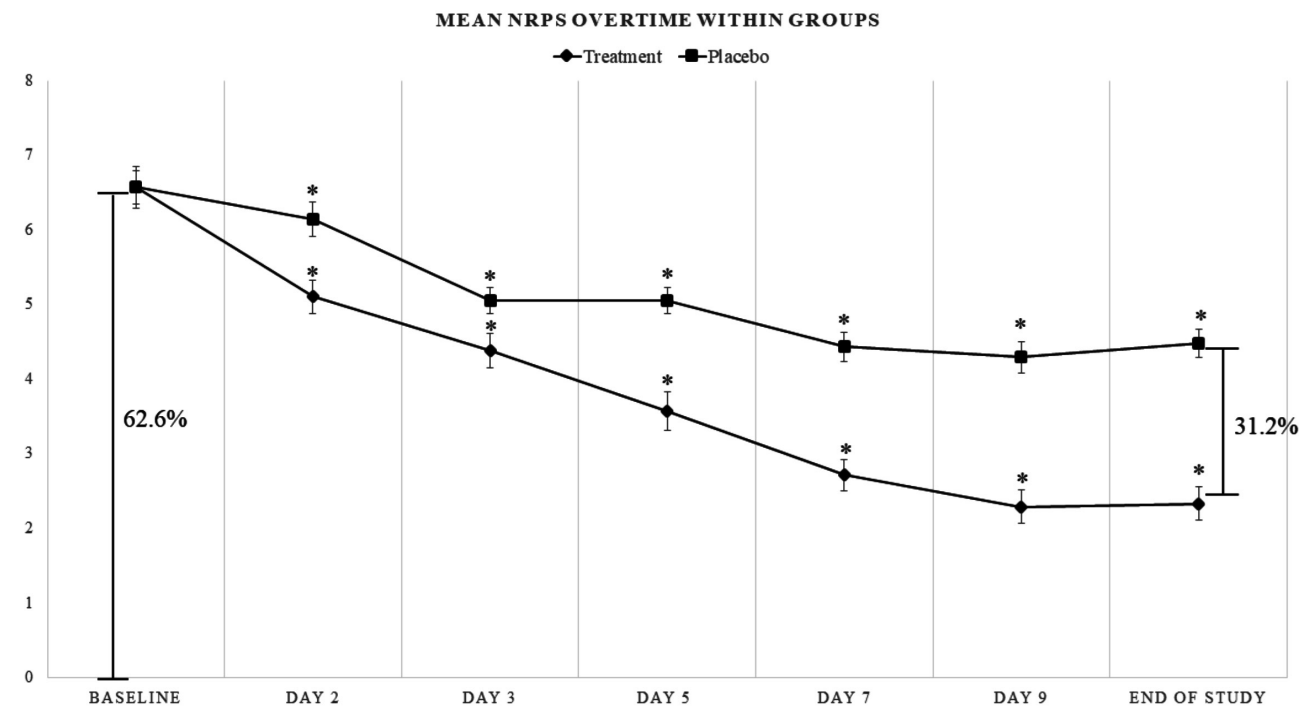

FIGURE 2. Main NPRS reduction across time

TABLE 2. Overall NPRS changes before and after intervention of both treatment groups

\begin{tabular}{|c|c|c|c|c|}
\hline Parameters & Treatment & Control & $95 \% \mathrm{Cl}$ & P value \\
\hline Mean NPRS of treatment vs. placebo over time (mean \pm SE) & $3.85 \pm 0.17$ & $5.14 \pm 0.17$ & $\begin{array}{c}3.49-4.21 \text { (treatment) } \\
4.79-5.50 \text { (placebo) }\end{array}$ & $0.001^{*}$ \\
\hline NPRS change of treatment vs. placebo over time (mean) & \multicolumn{2}{|c|}{-1.29} & $-1.84--0.75$ & $0.001^{*}$ \\
\hline NPRS reduction post- vs pre-treatment (mean $\pm S D]$ ) & $4.19 \pm 1.54$ & $2.1 \pm 0.83$ & $1.32-2.87$ & $0.001 *$ \\
\hline Percentage of NPRS reduction (mean \pm SD) & $62.6 \pm 17.47$ & $31.4 \pm 12.53$ & $\mathrm{~N} / \mathrm{A}$ & $0.001 *$ \\
\hline Nett percentage of NPRS reduction & \multicolumn{2}{|c|}{31.28} & $21.77-40.79$ & $0.001^{*}$ \\
\hline
\end{tabular}

*Significant at $p<0.05$

\section{DISCUSSION}

As many as 42 patients were recruited and allocated equally into each arm. All patients in both groups were compliant to the treatment regimen until completion of the study with zero drop out rate. This was probably due to the relatively brief intervention period that the patient had to attend. In general, patient's baseline characteristics were similar across two groups. Female predominated the study in both centers ( $62 \%$ and $57 \%$, respectively), similar to Dominguez et al. study [12], supporting the finding that female had a higher risk of developing DN [16]. Surprisingly, the mean duration of diabetes mellitus was relatively new, i.e. 4 years, but the complication of diabetic neuropathy can already be observed. This was contrary to the previous study, in which the average duration of DM was approximately 8 to 9 years $[9,17]$. This perhaps could be explained by the late onset of diagnosis, i.e. the patients might have already suffered from diabetes mellitus long before they were diagnosed. Accordingly, almost half of the patients diagnosed with DM had already suffered from peripheral neuropathic complication, inferring that the onset of DM might have had ocurred for a long period [18].

Baseline NPRS were similar between groups, both with a mean of 6.57. The corresponding score reflected moderate pain when referred to the functional performance. The pain intensity was comparable to that of other study findings, in which the average pain score was 5 to $6[19,20]$, but different to other two studies [21,22]. Repeated measures ANOVA test demonstrated that repeated examination across different time points within two groups demonstrated consistencies in therapeutic efficacy. It means that the continuously given treatment, regardless of the types of medications were able to ameliorate pain. Moreover, the improvements seen across all groups were consistent from one to another observational time point. It turned out that NPRS of the treatment group declined as soon as day 2 post intervention and continued to do so until the end of the study period, whereas placebo group demonstrated delayed effect and to a lesser extent than the former. In addition, the declining rate was consistent by -1.48 and -0.43-point in the beginning of intervention for treatment and placebo group, respectively, and continued to decline consistently until day 9, indicating that the given intervention can immediately ameliorate pain, hence act as a symptomatic reliever. However, treatment group demonstrated twice as much of NPRS decline rate with consistent statistical significance as opposed to placebo. The trend in both groups was only termi- 
nated after one week post treatment, wherein NPRS stopped declining further, yet did not experienced any increment or rebound phenomenon.

The underlying mechanism was not known yet, but we assumed due to the gradual decline of $\mathrm{MeCbl}$ concentration in the peripheral nerve, or more likely that the NPRS decline had already reached a plateau. Our observation period was not long enough to confirm this theory. Indeed, there are other studies with an extended observation period, ranging from 12 to 24 weeks. The efficacy results varied. Unfortunately, those studies did not monitor the symptoms (or pain intensity) from one to another time point as we did, ergo making it hard to detect any treatment wear-off.

In this study, we found that the treatment group experienced more significant NPRS reduction than the control group, both clinically and statistically. Treatment group had a statistically significant lower mean NPRS across all observational period when compared with placebo $(3.85 \pm 0.17$; $95 \%$ CI $3.49-$ 4.21 ; 5.14 \pm 0.17 ; $95 \%$ CI 4.79 - 5.50; $\mathrm{p}<0.001$, respectively). In fact, when we conducted a separate analysis which take into account only the pre- and post-intervention effect, we found NPRS reduction to more than twice among treatment when compared with placebo by then end of first week post intervention. Treatment group demonstrated NPRS reduction by $62.6 \%$ from baseline, which was almost twice as much as those of the control group (i.e. $31.4 \%$; $\mathrm{p}<0.001$ ). This finding was consistent with the previous study [23], in which similar treatment with oral amitryptiline and intravenous $\mathrm{MeC}$ bl versus oral amitryptiline with intravenous aquabidest injection administered for the same period of time was able to significantly reduce NPRS score by 79 and $48 \%$, respectively. In this study, we obtained a lower pain reduction rate when compared to those of other studies [21-23], yet still fulfilled the generally acceptable criteria for a successful treatment for pain, i.e. if the pain can be reduced to at least $50 \%$ or more [24].

To date, there has been a minimal number of studies specifically evaluating the efficacy of $\mathrm{MeCbl}$ in painful diabetic neuropathy cases. One study employed oral supplement in which $2 \mathrm{mg}$ of MeCbl was among one of the constituents, given for 24 weeks [25]. A significant symptomatic relief was achieved among treatment group when compared with placebo. However, the study used orally ingested MeCbl which was known to have lower bioavailability when compared with intravenous injection. In addition, the study also did not distinguish between painless and PDN which to date was known to have multiple differences in pathophysiology, types of nerve involved, and risk factors [26]. Another study used MeCbl for the treatment of DN, yet again, did not specifically target those with PDN and did not compare the results with placebo [9].

Furthermore, our study demonstrated a significant reduction in pain scale for as early as the second day post treatment. Although most of this reduction could be accounted for the amitryptiline, the second surge of NPRS decline at day 7 post treatment was probably due to MeCbl. This was interesting since in the previous studies, treatment only began to ameliorate pain at day 14 . The widely believed theory is that $\mathrm{MeCbl}$ enchances nerve myelination in order to ameliorate pain. However, given the account that DN usually affects $C$ fibers which lack myelin, the acute improvement seen in our study was potentially due to pleiotropic effects of $\mathrm{MeCbl}$, including promoting axonal regeneration, protecting against glutamate-induced neurotoxicity, as well as inhibiting ectopic spontaneous discharge [7,11].

We also used intravenous injection which may affect practicality and feasibility of MeCbl delivery among subjects. We did this to achieve maximum plasma and intracellular concentration to address the relatively brief treatment period (i.e. 9 days vs. several weeks in other studies). Our study results may therefore be extrapolated using alternative delivery approach, including intramuscular and per oral treatment.

Regardless, given the role of $\mathrm{MeCbl}$ in the pathophysiology of DN, continuous treatment might be more effective in ameliorating as well as sustaining PDN. This study encourages other similar studies in the future with larger patient numbers and longer observational period to better profile the clinical efficacy and sustainability of $\mathrm{MeCbl}$ in ameliorating PDN. Lastly, MeCbl administration was relatively safe with no serious side effects thereof. In conclusion, treatment with oral amitryptiline in conjunction with intravenous $\mathrm{MeCbl}$ was significantly more effective in ameliorating PDN than with oral amitriptyline and saline injection with no serious adverse effects.

\section{CONCLUSIONS}

Intravenous $\mathrm{MeCbl}$ was proven to be a safe and significantly more effective adjunct treatment in relieving PDN. The therapeutic effect can also be achieved in an immediate fashion.

\section{Note}

All authors contributed equally in this study. 


\section{REFERENCES}

1. Peltier A, Goutman SA, Callaghan BC. Painful diabetic neuropathy. BMJ. 2014;348:g1799.

2. Tesfaye S, Boulton AJ, Dyck PJ, Freeman R, Horowitz M, Kempler P et al. Diabetic neuropathies: update on definitions, diagnostic criteria, estimation of severity, and treatments. Diabetes Care. 2010;33(10):2285-93.

3. Javed S, Petropoulos IN, Alam U, Malik RA. Treatment of painful diabetic neuropathy. Ther Adv Chronic Dis. 2015;6(1):15-28.

4. D’Amato C, Morganti R, Greco C, Di Gennaro F, Cacciotti L, Longo S et al. Diabetic peripheral neuropathic pain is a stronger predictor of depression than other diabetic complications and comorbidities. Diab Vasc Dis Res. 2016;13(6):418-28.

5. Jain R, Jain S, Raison CL, Maletic V. Painful diabetic neuropathy is more than pain alone: examining the role of anxiety and depression as mediators and complicators. Curr Diab Rep. 2011;11(4):275-84.

6. Van Acker K, Bouhassira D, De Bacquer D, Weiss S, Matthys K, Raemen $H$ et al. Prevalence and impact on quality of life of peripheral neuropathy with or without neuropathic pain in type 1 and type 2 diabetic patients attending hospital outpatients clinics. Diabetes Metab. 2009;35(3):206-13.

7. Zhang M, Han W, Hu S, Xu H. Methylcobalamin: a potential vitamin of pain killer. Neural Plast. 2013;2013:424651.

8. Ide H, Fujiya S, Asanuma Y, Tsuji M, Sakai H, Agishi Y. Clinical usefulness of intrathecal injection of methylcobalamin in patients with diabetic neuropathy. Clin Ther. 1987;9(2):183-92.

9. Yaqub BA, Siddique A, Sulimani R. Effects of methylcobalamin on diabetic neuropathy. Clin Neurol Neurosurg. 1992;94(2):105-11.

10. Xu J, Wang W, Zhong XX, Feng Y, Wei X, Liu XG. EXPRESS: Methylcobalamin ameliorates neuropathic pain induced by vincristine in rats: Effect on loss of peripheral nerve fibers and imbalance of cytokines in the spinal dorsal horn. Mol Pain. 2016;12.

11. Chaplan SR, Guo HQ, Lee DH, Luo L, Liu C, Kuei C et al. Neuronal hyperpolarization-activated pacemaker channels drive neuropathic pain. J Neurosci. 2003;23(4):1169-78.

12. Dominguez J, Ng A, Damian L. A prospective, open label, 24-week trial of methylcobalamin in the treatment of diabetic polyneuropathy. Journal of Diabetes Mellitus. 2012;2(4):4.

13. Talaei A, Siavash M, Majidi H, Chehrei A. Vitamin B12 may be more effective than nortriptyline in improving painful diabetic neuropathy. Int J Food Sci Nutr. 2009;60 Suppl 5:71-6.

14. Schulz KF, Altman DG, Moher D. CONSORT 2010 Statement: updated guidelines for reporting parallel group randomised trials. BMJ. 2010;340:c332.
15. Spallone V, Morganti R, D'Amato C, Greco C, Cacciotti L, Marfia GA. Validation of DN4 as a screening tool for neuropathic pain in painful diabetic polyneuropathy. Diabet Med. 2012;29(5):578-85.

16. Abbott CA, Malik RA, van Ross ER, Kulkarni J, Boulton AJ. Prevalence and characteristics of painful diabetic neuropathy in a large community-based diabetic population in the U.K. Diabetes Care. 2011;34(10):2220-4.

17. Davies M, Brophy S, Williams R, Taylor A. The prevalence, severity, and impact of painful diabetic peripheral neuropathy in type 2 diabetes. Diabetes Care. 2006;29(7):1518-22.

18. Spijkerman AM, Dekker JM, Nijpels G, Adriaanse MC, Kostense PJ, Ruwaard $D$ et al. Microvascular complications at time of diagnosis of type 2 diabetes are similar among diabetic patients detected by targeted screening and patients newly diagnosed in general practice: the hoorn screening study. Diabetes Care. 2003;26(9):2604-8.

19. Boonstra AM, Stewart RE, Koke AJ, Oosterwijk RF, Swaan JL, Schreurs KM et al. Cut-Off Points for Mild, Moderate, and Severe Pain on the Numeric Rating Scale for Pain in Patients with Chronic Musculoskeletal Pain: Variability and Influence of Sex and Catastrophizing. Front Psychol. 2016;7:1466.

20. Gore M, Brandenburg NA, Dukes E, Hoffman DL, Tai KS, Stacey B. Pain severity in diabetic peripheral neuropathy is associated with patient functioning, symptom levels of anxiety and depression, and sleep. J Pain Symptom Manage. 2005;30(4):374-85.

21. Dongre YU, Swami OC. Sustained-release pregabalin with methylcobalamin in neuropathic pain: an Indian real-life experience. Int J Gen Med. 2013;6:413-7.

22. Khan $A$, Tao $S$, Feng W. The efficacy and safety of fixed dose sustained-release pregabalin, aceclofenic and methylcobalamin in sciatica and peripheral neuropathic pain. World Journal of Pharmaceutical Research. 2015;4(8):9.

23. Purwata T, Rudy M, Putra P, Nuartha A, Samatra D, Laksmidewi A et al. 6th International Congress on Neuropathic Pain. Gothenburg, Sweden: NeuPSIG2017.

24. Schreiber AK, Nones CF, Reis RC, Chichorro JG, Cunha JM. Diabetic neuropathic pain: Physiopathology and treatment. World J Diabetes. 2015;6(3):432-44.

25. Fonseca VA, Lavery LA, Thethi TK, Daoud Y, DeSouza C, Ovalle F et al. Metanx in type 2 diabetes with peripheral neuropathy: a randomized trial. Am J Med. 2013;126(2):141-9.

26. Spallone V, Greco C. Painful and painless diabetic neuropathy: one disease or two? Curr Diab Rep. 2013;13(4):533-49. 